\title{
Carotid ultrasound investigation as a prognostic tool for patients with diabetes mellitus
}

Matthias Hoke ${ }^{1}$, Martin Schillinger ${ }^{1}$, Erich Minar ${ }^{1}$, Georg Goliasch², Christoph J. Binder ${ }^{3}$ and Florian J. Mayer ${ }^{3^{*}}$ (D)

\begin{abstract}
Background: Experimental and clinical data indicate a major influence of diabetes on atherogenesis. We aimed to assess whether the effect of diabetes on long-term mortality in asymptomatic patient with carotid stenosis is contingent upon the degree of the carotid atherosclerotic burden.

Methods: 1065 patients with neurological asymptomatic carotid atherosclerosis as evaluated by duplex sonography were prospectively followed for cause-specific mortality.

Results: During a median of 11.8 years, a total of 549 deaths, including 362 cardiovascular deaths, were recorded. Diabetes and glycohemoglobin A1c (Hba1c) levels were significantly associated with mortality. Diabetes displayed an independent risk for all-cause (adjusted HR 1.62; 95\% Cl 1.35-1.94) and cardiovascular death (adjusted HR 1.75, 95\% Cl 1.40-2.19). The adjusted hazard ratio per increase of 1\% of Hba1c levels was $1.21(\mathrm{P}<0.01)$ for all-cause and $1.31(P<0.01)$ for cardiovascular mortality, respectively. Patients with diabetes mellitus and a higher degree of carotid stenosis and were at great risk of adverse outcome. Only $21 \%$ of the asymptomatic diabetic patients with carotid narrowing over $50 \%$ survived, whereas $62 \%$ of the patients without diabetes and with carotid atherosclerosis below 50\% were still alive after 12-years of follow-up. The high risk for all-cause and cardiovascular death of these patients remained significant after adjustment for various established cardiovascular risk factors in multivariable regression analysis (adjusted hazard ratio 2.4, $\mathrm{P}<0.001$; compared to patients without diabetes and $<50 \%$ carotid atherosclerosis).

Conclusion: Diabetic patients with carotid stenosis $\geq 50 \%$ are at exceptional high risk for all-cause and cardiovascular death. Thus, routinely ultrasound investigation of the carotid arteries might be a valuable prognostic tool for patients with diabetes mellitus.
\end{abstract}

Keywords: Carotid atherosclerosis, Diabetes mellitus, Hba1c, Biomarker, Risk factor

\section{Background}

Diabetes mellitus is associated with increased morbidity and mortality, and is linked to some acute but mainly chronic complications, based on functional and structural damages to the blood vessels. The disease is, unsurprisingly, a major risk factor for cardiovascular disease and a plethora of experimental and clinical studies link

\footnotetext{
*Correspondence: florian.mayer@meduniwien.ac.at

${ }^{3}$ Department of Laboratory Medicine, Medical University of Vienna,

Währinger Gürtel 18-20, 1090 Vienna, Austria

Full list of author information is available at the end of the article
}

hyperglycemia to the development and progression of atherosclerosis. Various mechanisms by which diabetes contributes to cardiovascular disease and atherosclerosis have been identified. Alterations of the vessel wall, due to endothelial and smooth muscle cell dysfunction, are the main characteristics of diabetic vasculopathy. The chronic hyperglycemic state increases the generation of advanced glycation end products (AGEs), which are formed by sequential non-enzymatic reactions of glucose and other glycating compounds. When glucose mediates the reaction, initially the Amadori adduct fructosyl-lysine is formed [1]. In hemoglobin, this adduct is 
called glycohemoglobin A1c (HbA1c), which standardized glycemic control in diabetic patients. AGEs cause the development of reactive oxygen species, which leads to lipid peroxidation and generation of oxidized free fatty acids, major contributors to the development of endothelial dysfunction and atherosclerosis [2]. The data of these experimental studies are well reflected in observational studies as well as in clinical trials of patients with diabetes and atherosclerotic diseases. Today, diabetes is recognized as a major risk factor for coronary artery disease, peripheral arterial disease and cerebrovascular disease [3-5]. Even though there is plenty of data available about associations between clinical manifestations of symptomatic atherosclerotic diseases (e.g. myocardial infarction) and diabetes mellitus, there is still a lack of understanding how diabetes affects the long-term outcome in patients with subclinical atherosclerosis. Some clinical studies investigated the relationship of specific sonographic characteristics of the carotid arteries as well as the short term clinical outcome of patients with carotid atherosclerosis and diabetes $[6,7]$.

Similar to diabetes, atherosclerosis progresses over decades of life-time and frequently remains asymptomatic and undiagnosed up to the occurrence of a first clinical event. The role of diabetes in this context has not been sufficiently characterized. The aim of this study was to evaluate whether the degree of carotid stenosis and diabetes mellitus jointly predict long-term mortality in asymptomatic patients with carotid atherosclerosis.

\section{Methods}

In this single-center study, we prospectively enrolled 1363 consecutive patients who underwent ultrasound investigations of the extracranial carotid arteries between March 2002 and March 2003. Study design, inclusion, and exclusion criteria have been published previously [8]. Patients with prevalent atherosclerotic carotid artery disease, defined by the presence of non-stenotic plaques or carotid stenosis of any degree that were neurologically asymptomatic at the time of screening, were enrolled. The main indications for performing ultrasound investigation were carotid bruits, prevalence of cardiovascular risk factors, and known atherosclerotic diseases in other vessel areas. Patients with current infectious, inflammatory diseases or active malignancies, symptomatic of carotid artery disease that necessitated revascularization therapy, patients having undergone bilateral carotid occlusions, bilateral stent implantation, or bilateral carotid endarterectomy, as well as patients with a myocardial infarction, stroke, coronary revascularization, or peripheral vascular surgery during the preceding 6 months, were excluded from the study. Cardiovascular and all-cause mortality were assessed by searching the national death register for the specific cause of death (according to the International Statistical Classification of Diseases and Related Health Problems, 10th Revision). Only the specific cause of death (e.g., acute myocardial infarction) was used to categorize death as either all-cause, cardiovascular, or non-cardiovascular death. In $43 \%$ of deaths, the underlying cause was assessed by autopsy.

\section{Clinical and laboratory data}

Every enrolled patient completed a detailed study questionnaire that was reviewed by a physician assessing the patient's medical history, current medication, biometric data, and family history. All clinical characteristics were ascertained by 2 independent observers. Antecubital venous blood samples were drawn and analyzed directly without freezing according to local laboratory standard procedures within 2 to $4 \mathrm{~h}$ of sampling. Serum levels of Hbalc were determined at admission (DADE Behring, IL, USA). Treating physicians and ultrasonographers were blinded for all laboratory values, color-coded duplex sonography, and grading of internal carotid artery stenosis.

\section{Degree of carotid stenosis}

Duplex examinations at baseline were performed on an Acuson 128 XP10 with a $7.5-\mathrm{MHz}$ linear array probe (Acuson, Malvern, PA). The degree of carotid artery narrowing was obtained according to 6 categories, corresponding to NASCET (North American Symptomatic Carotid Endarterectomy Trial) angiographic graduation $[8,9]$. A cut-off at $50 \%$ degree of carotid narrowing, measured with ultrasound sonography, was set to obtain clinically useful measures for the effect sizes. The rationale behind this was that carotid narrowing (assessed by sonography) less than $50 \%$ is generally considered hemodynamically insignificant $[9,10]$. Our interobserver agreement was adequate with respect to the absolute degree of stenosis ( $\kappa=0.83,95 \%$ CI 0.79 to 0.88$)$ and with respect to progression of the disease $(\kappa=0.85,95 \%$ CI 0.80 to 0.89 ).

\section{Definitions}

Definitions of risk factors and comorbidities were published previously [8]. Briefly, hypertension was considered present in patients with blood pressure above $140 / 90 \mathrm{~mm} \mathrm{Hg}$ or in patients taking antihypertensive medication. A family history of atherosclerotic disease was considered positive if its presence had been verified in a first-degree relative.

Since patients were enrolled between 2002 and 2003, diabetes mellitus was defined as fasting blood glucose levels $>125 \mathrm{mg} / \mathrm{dL}$ (i.e. $>7.0 \mathrm{mmol} / \mathrm{L}$ ) according to the 1997 criteria of the American Diabetes Association [11]. 
In addition, according to the 2019 classification of diabetes of the American Diabetes Association, patients with baseline glycohemoglobin A1c levels $\geq 6.5 \%$ were retrospectively also defined as diabetic [12].

\section{Statistical methods}

Continuous data are presented as median and interquartile range (range from the 25th to the 75th percentile). Discrete data are given as counts and percentages. Analysis of variance and the $\mathrm{X}^{2}$ test were used for comparisons between, as appropriate. The log-rank test was used for comparison between groups. Event-free survival probabilities were estimated using the Kaplan-Meier method. Univariable and multivariable Cox proportional hazards models were applied to assess the association between diabetes mellitus, Hba1c, degree of carotid stenosis and the occurrence of either all-cause or cardiovascular death. The following variables were included as co-variables in every multivariable model: age (years), sex (male/ female), history of myocardial infarction (binary), history of stroke (binary), peripheral arterial disease (binary), body mass index $\left(\mathrm{kg} / \mathrm{m}^{2}\right)$, hypertension (binary), serum creatinine $(\mathrm{mg} / \mathrm{dL})$, levels of triglycerides $(\mathrm{mg} / \mathrm{dL})$, total cholesterol levels $(\mathrm{mg} / \mathrm{dL})$, low density lipoprotein cholesterol levels $(\mathrm{mg} / \mathrm{dL})$, high sensitive C-reactive protein $(\mathrm{mg} / \mathrm{dL})$ and statin treatment (binary). The selection of the variables was defined a priori and is based on current guidelines for cardiovascular risk prediction. All of the variables listed above were included in every multivariable Cox proportional hazard model used for this study. Results of the Cox models are presented as hazard ratios (HR; 95\% confidence interval [CI]). We assessed the overall model fit using Cox-Snell residuals. We also tested the proportional hazard assumption for all covariates using Schoenfeld residuals (overall test) and the scaled Schoenfeld residuals (variable-by-variable testing).

An improvement in individual risk prediction was examined using the net reclassification improvement [13]. Interactions between plasma levels of Hba1c or diabetes mellitus (binary) and degree of carotid artery stenosis were tested by entering interaction terms in the Cox proportional hazard regression models. A 2 -sided $P$ value of $<0.05$ was considered significant. All calculations were performed with SPSS (version 20.0, SPSS Inc.) and the STATA11 software package (Stata Corp.) for Windows.

\section{Results}

\section{Study population}

A total of 1363 patients were enrolled in the study. Ninety-five (7\%) of these patients had missing duplex ultrasound follow-up data, and 203 patients (16\%) were lost to clinical follow-up, leaving 1065 patients for the final analysis. The 298 patients who had to be excluded did not significantly differ from the patients who were included in terms of baseline and demographic parameters (data not shown).

In total, 1065 patients were included in the final analysis. The median age was 69 years (IQR 61-76 years) at the time of inclusion and $668(62.7 \%)$ were male (Table 1). Within a period of 11.9 years (IQR 6.0-12.4 years), we recorded $548(51.5 \%)$ deaths from any cause. Of these, 367 patients (67\%) died from cardiovascular causes, 142 (13.3\%) of malignant diseases, and 45 (4.2\%) of other causes.

\section{Diabetes and long-term mortality}

Diabetes mellitus was present in 335 (31.5\%) subjects. The vast majority suffered from type 2 diabetes (95.3\%). During follow up, 69.8\% (169) of patients with prevalent diabetes at enrollment died compared to $46.1 \%$ (379) of patients without diabetes (Table 2). The all-cause mortality rate was $66.9 \%$ in diabetic and $44.4 \%$ in non-diabetic patients, and the cardiovascular mortality rate was $47.5 \%$ in diabetic and $28.5 \%$ in non-diabetic patients, respectively $(\mathrm{P}<0.01)$. In multivariable analyses diabetes displayed a robust and independent risk for all-cause (adjusted HR 1.62, 95\% CI 1.35-1.94) and cardiovascular death (adjusted HR 1.75, 95\% CI 1.40-2.19). Glycohemoglobin A1c levels were significantly associated with mortality. The median HbA1c was 6.0\% (IQR 5.6-6.6\%) in the overall and $7.2 \%$ (IQR $6.4-8.1 \%$ ) in the diabetic population and the risk of all-cause and cardiovascular mortality significantly increased in patients with elevated serum levels of Hbalc. The adjusted hazard ratio per increase of $1 \%$ of Hba1c levels was 1.21 (CI 1.12-1.32, $\mathrm{P}<0.01$ ) for all-cause and 1.30 (CI 1.20-1.43, $\mathrm{P}<0.01$ ) for cardiovascular mortality, respectively (Table 2 ).

\section{Diabetes and degree of carotid stenosis}

377 (35.4\%) patients had unilateral or bilateral carotid artery narrowing of $\geq 50 \%$ at enrollment. To assess the joint effect of diabetes and carotid stenosis on long-term outcome, the patient population was stratified into 4 groups according to the degree of carotid narrowing and the frequency of diabetes. Group 1 were non-diabetic patients with carotid narrowing $<50 \%$. Group 2 included diabetic patients with carotid narrowing $<50 \%$. Group 3 represented patients with carotid narrowing $\geq 50 \%$ but without diabetes, and group 4 patients with both carotid stenosis $\geq 50 \%$ and diabetes.

The cumulative 12-year survival rates in groups 1 to 4 were $62 \%, 43 \%, 40 \%$, and $21 \%$ for all-cause death and $76 \%, 63 \%, 57 \%$, and $44 \%$ for cardiovascular death $(\mathrm{P}<0.01$; Fig. 1) Adjusted HRs for the risk of all-cause death in groups 2 to 4 were 1.27 (CI 1.01-1.59), 1.56 (CI $1.22-1.99$ ), and 2.22 (CI 1.71-2.99; $\mathrm{P}<0.01$ ), and for 
Table 1 Baseline characteristics and risk factors of 1065 patient

\begin{tabular}{|c|c|c|c|c|c|}
\hline \multirow[t]{2}{*}{ Variable } & \multicolumn{4}{|l|}{ Combined model } & \multirow[t]{2}{*}{ P-value } \\
\hline & $\begin{array}{l}\text { Carotid } \\
\text { stenosis }<50 \% \\
\text { and non-diabetic } \\
n=486\end{array}$ & $\begin{array}{l}\text { Carotid } \\
\text { stenosis } \geq 50 \% \\
\text { and non-diabetic } \\
n=247\end{array}$ & $\begin{array}{l}\text { Carotid } \\
\text { stenosis }<50 \% \\
\text { and diabetes } \\
n=205\end{array}$ & $\begin{array}{l}\text { Carotid } \\
\text { stenosis } \geq 50 \% \\
\text { and diabetes } \\
n=127\end{array}$ & \\
\hline Age (years) & $66.9(59.0-75.3)$ & $71.9(64.1-77.5)$ & $68.6(61.2-75.0)$ & $70.9(63.5-77.7)$ & $<0.01$ \\
\hline Male (binary) & $290(59.7)$ & $158(64.0)$ & $138(67.3)$ & $82(64.6)$ & 0.25 \\
\hline History of PAD (binary) & $155(31.9)$ & $116(47.0)$ & $108(52.7)$ & $77(60.6)$ & $<0.01$ \\
\hline History of Ml (binary) & $90(18.5)$ & $48(19.4)$ & $62(30.2)$ & $57(44.9)$ & $<0.01$ \\
\hline History of Stroke (binary) & $68(14.0)$ & $51(20.6)$ & $24(11.7)$ & $33(26.0)$ & $<0.01$ \\
\hline Art. Hypertension (binary) & $291(59.9)$ & $177(71.7)$ & $158(77.1)$ & $105(82.7)$ & $<0.01$ \\
\hline Current Smoker (binary) & $124(25.5)$ & $72(29.1)$ & $48(23.4)$ & $43(33.9)$ & 0.14 \\
\hline Family history of atherosclerosis (binary) & $252(51.9)$ & $152(61.5)$ & $105(51.2)$ & $81(63.8)$ & 0.01 \\
\hline BMI (ratio) & $25.6(23.7-28.1)$ & $26.2(23.7-28.3)$ & $27.7(30.5-24.7)$ & $26.8(24.2-29.6)$ & $<0.01$ \\
\hline $\mathrm{HbA1c}(\%)$ & $5.7(5.4-6)$ & $5.9(5.6-6.1)$ & $7.1(6.6-7.9)$ & $7.1(6.6-7.8)$ & $<0.01$ \\
\hline Total cholesterol (mg/dl) & $207(178-243)$ & $208(181-237)$ & $194(169-221)$ & $202(170-223)$ & 0.01 \\
\hline Triglycerides (mg/dl) & $139(99-201)$ & $147(109-205)$ & $154(111-235)$ & $165(115-237)$ & $<0.01$ \\
\hline HDL cholesterol (mg/dl) & $53(43-63)$ & $50(42-60)$ & $45(39-55)$ & $46(38-54)$ & $<0.01$ \\
\hline LDL cholesterol (mg/dl) & $122(95-151)$ & $122(97-146)$ & $109(87-137)$ & $115(92-138)$ & $<0.01$ \\
\hline hs-CRP (mg/dl) & $0.25(0.11-0.58)$ & $0.29(0.13-0.68)$ & $0.33(0.19-0.66)$ & $0.35(0.15-0.79)$ & $<0.01$ \\
\hline Serum creatinine (mg/dl) & $1.02(0.92-1.18)$ & $1.07(0.96-1.21)$ & $1.08(0.95-2.18)$ & $1.13(0.97-1.30)$ & 0.02 \\
\hline Statins (binary) & $247(50.8)$ & $161(65.2)$ & $126(61.5)$ & $86(67.7)$ & $<0.01$ \\
\hline Insulin therapy \pm Oral antidiabetics (binary) & - & - & $47(22.9)$ & $27(21.3)$ & $0.53^{*}$ \\
\hline Oral antidiabetics (binary) & - & - & $101(49.3)$ & $65(51.2)$ & $0.13^{*}$ \\
\hline
\end{tabular}

Continuous data are presented as the median and the interquartile range. Discrete data are given as counts and percentages

* P-value for the comparison between the groups "Carotid stenosis $<50 \%$ and diabetes" and "Carotid stenosis $\geq 50 \%$ and diabetes"

cardiovascular death 1.26 (CI 0.96-1.64), 1.63 (CI 1.201.2.21), and 2.40 (CI 1.72-3.34; $\mathrm{P}<0.01)$, compared with the first group (Table 2).

Improvement in individual risk stratification with combined assessment of degree of carotid stenosis and diabetes mellitus was confirmed by a significant improvement in the net reclassification improvement, with $39 \%$ $( \pm 6 \% ; \mathrm{P}<0.001)$ for all-cause and $37 \%( \pm 6 \% ; \mathrm{P}<0.001)$ for cardiovascular mortality, respectively, as compared with degree of carotid stenosis alone. We did not observe any significant interactions between diabetes or Hba1c, degree of carotid artery stenosis and mortality ( $\mathrm{P}$-value for interaction with diabetes $=0.64$, and with Hbalc $\geq 50 \%=0.39$ ).

\section{Discussion}

Our study found a clear association between the presence of carotid atherosclerosis and mortality among diabetic patients. In a combined assessment, we identified that subjects with diabetes and asymptomatic carotid stenosis $\geq 50 \%$ are at an exceptional high risk for adverse outcome. If a patient at time of inclusion was diagnosed with both diabetes mellitus and carotid stenosis above $50 \%$, the patient had only a $\sim 20 \%$ chance to survive the following 12 years. These findings are more than alarming, considering that the whole cohort had a survival rate of $\sim 50 \%$. Further, diabetic patients with carotid stenosis $\geq 50 \%$ had a near 2.5 -fold increase in risk of cardiovascular death compared to non-diabetic patients with carotid atherosclerosis below 50\% narrowing. These latter findings were independent of various established cardiovascular risk factors or previous cerebro- or cardiovascular events. We further identified that combining carotid stenosis with diabetes mellitus improved the risk stratification by near $40 \%$ for the risk of all-cause and cardiovascular death, respectively. In contrast, the clinical diagnosis diabetes mellitus, based on hbalc and serum glucose levels alone, displayed a highly significant, but rather weak association with (cardiovascular) mortality in patients in asymptomatic carotid atherosclerosis after 12-years of follow-up.

\section{Diabetes, atherogenesis and cardiovascular outcome}

The association between diabetes and the development of cardiovascular disease has been well established in the last decades or so [3-5]. More recently, it has been shown that patients with late onset autoimmune diabetes in the adult (LADA) are at increased macrovascular risk 
Table 2 Results of univariable and multivariabe Cox regression analyses

\begin{tabular}{|c|c|c|c|c|c|c|c|}
\hline \multirow[t]{2}{*}{ Variable } & & \multicolumn{3}{|c|}{ All-cause mortality } & \multicolumn{3}{|c|}{ Cardiovascular mortality } \\
\hline & & Hazard ratio & $\mathrm{Cl}$ & P-value & Hazard ratio & $\mathrm{Cl}$ & P-value \\
\hline Univariable & $\mathrm{n}$ & & & & & & \\
\hline Diabetes mellitus & 335 & 1.14 & $1.06-1.22$ & $<0.001$ & 1.14 & $1.08-1.22$ & $<0.001$ \\
\hline Per increase of $1 \%$ of hba $1 c$ & 1065 & 1.23 & $1.15-1.32$ & $<0.001$ & 1.30 & $1.20-1.41$ & $<0.001$ \\
\hline Carotid stenosis $\geq 50 \%$ & 374 & 1.67 & $1.40-1.97$ & $<0.001$ & 1.70 & $1.39-2.08$ & $<0.001$ \\
\hline \multicolumn{8}{|l|}{ Combined model } \\
\hline Carotid stenosis $<50 \% ; \varnothing \mathrm{DM}^{a}$ & 522 & Ref & & & & & \\
\hline Carotid stenosis $\geq 50 \% ; \varnothing \mathrm{DM}$ & 276 & 1.65 & $1.33-2.06$ & $<0.001$ & 1.71 & $1.30-2.25$ & $<0.001$ \\
\hline Carotid stenosis < 50\%; DM & 166 & 1.86 & $1.48-2.34$ & $<0.001$ & 2.13 & $1.61-2.81$ & $<0.001$ \\
\hline Carotid stenosis $\geq 50 \%$; DM & 101 & 3.23 & $2.53-4.13$ & $<0.001$ & 3.67 & $2.73-4.94$ & $<0.001$ \\
\hline \multicolumn{8}{|l|}{ Multivariable } \\
\hline Diabetes mellitus & 335 & 1.14 & $1.06-1.22$ & $<0.001$ & 1.14 & $1.08-1.22$ & $<0.001$ \\
\hline Per increase of $1 \%$ of hba $1 \mathrm{c}$ & 1065 & 1.21 & $1.12-1.32$ & $<0.001$ & 1.30 & $1.20-1.43$ & $<0.001$ \\
\hline Carotid stenosis $\geq 50 \%$ & 374 & 1.28 & 1.30 & 0.004 & 1.28 & $1.03-1.60$ & 0.025 \\
\hline \multicolumn{8}{|l|}{ Combined model } \\
\hline Carotid stenosis $<50 \% ; \varnothing \mathrm{DM}^{\mathrm{a}}$ & 522 & Ref & & & & & \\
\hline Carotid stenosis $\geq 50 \% ; \varnothing \mathrm{DM}$ & 276 & 1.27 & $1.01-1.59$ & 0.040 & 1.26 & $0.96-1.64$ & 0.072 \\
\hline Carotid stenosis < 50\%; DM & 166 & 1.56 & $1.22-1.99$ & $<0.001$ & 1.78 & $1.33-2.39$ & $<0.001$ \\
\hline Carotid stenosis $\geq 50 \%$; DM & 101 & 2.22 & $1.71-2.99$ & $<0.001$ & 2.35 & $1.71-3.24$ & $<0.001$ \\
\hline
\end{tabular}

Cl confidence interval, $H R$ hazard ratio, hsCRP high sensitivity $C$-reactive protein

a Reference category; adjusted for age, sex, body mass index, hypertension smoking, history of peripheral artery disease, history of stroke history of myocardial infarction, low-density lipoprotein cholesterol levels, triglyceride levels, statin treatment, serum creatinine, hsCRP

despite a "healthier" risk profile as compared to patients with type 2 diabetes [14]. It appears, that especially in "younger" populations inflammation and plaques composition are main triggers for adverse outcome, since atherosclerotic plaques with thin fibrous caps and large necrotic cores are clearly associated with cardiovascular events and death [15]. In this context, fibrinogen was recently found to be inversely associated with intraplaque hemorrhage- and necrotic core-volume, independently of inflammation [16]. Fernández-Friera et al. lately described that arterial inflammation is highly prevalent in middle-aged individuals with known subclinical atherosclerosis, and thus may trigger early cardiovascular events [17].

Our results are in line with previous outcome studies which predominantly investigated the role of carotid intima media thickness in patients at cardiovascular risk [18-22]. There have also been reports that information about the presence of carotid atherosclerosis can be used for the prediction of outcome in patient with coronary artery disease [23] and that the combined assessment of IMT and interadventitia common carotid artery diameter is useful to stratify a patient's individual cardiovascular risk profile [24]. However, our study oversees a 12-year period of follow-up and provides robust data on long term outcome in patients with prevalent or advanced carotid atherosclerosis among a well-defined cohort.

Diabetes as well as carotid atherosclerosis are frequently referred to as "silent killers" and our study not only confirms, but also emphasizes the strong additive effect of both risk factors. The prevalence of carotid atherosclerosis in patients older than 60 years is high and the same holds true for diabetes $[25,26]$. Based on our data, we believe that a screening method for subclinical atherosclerosis in patients with diabetes might be a useful tool for risk stratification. According to the current guidelines of the American Diabetes Association for Cardiovascular Disease and Risk Management, screening for coronary artery disease in asymptomatic patients with diabetes mellitus and a high risk profile for cardiovascular disease, is currently not recommended [27]. This is mainly attributed to the screening methods for coronary artery disease which are costly, often risky for the patient, timeconsuming and require certain infrastructure. On the contrary, the performance of an ultrasound investigation of the carotid arteries is simple, cost-effective and does not pose any risk to the patient.

Anti-diabetic medication and cardiovascular outcome Various novel anti-diabetic drugs such as SodiumGlucose Co-Transporter 2 (SGLT2) inhibitors or 


\section{a All-Cause Mortality}

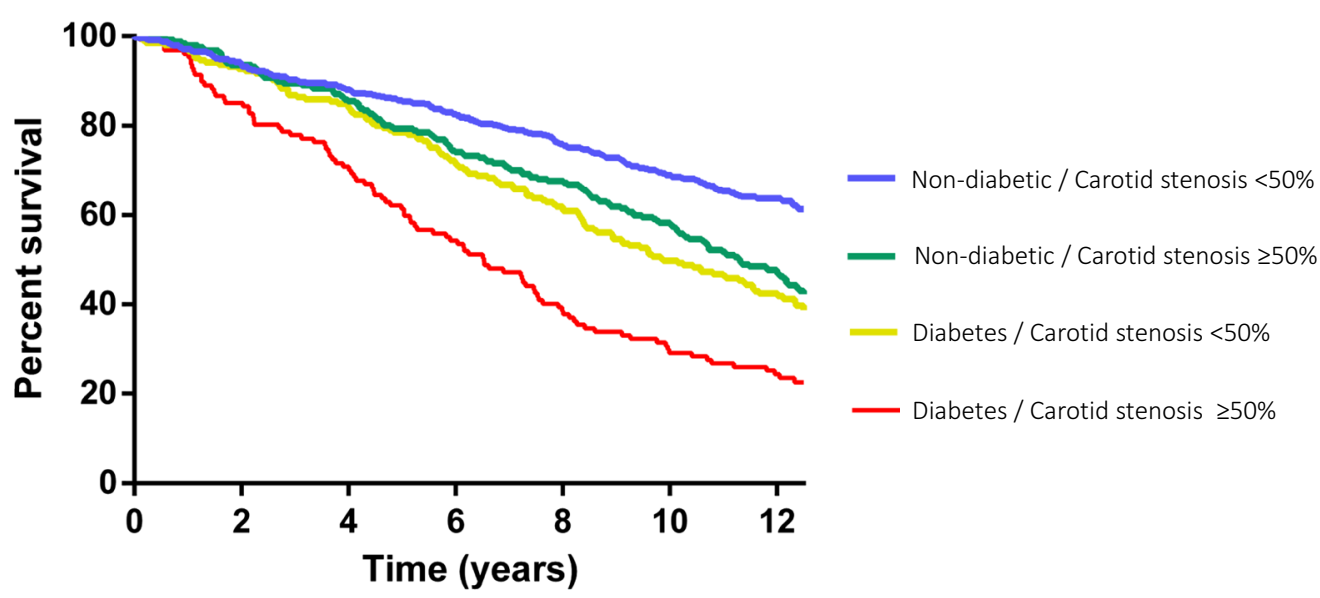

\begin{tabular}{|c|c|c|c|c|c|}
\hline \multicolumn{6}{|c|}{ No. at risk } \\
\hline $1^{\text {st }}$ & group & 486 & 428 & 369 & 244 \\
\hline $2^{\text {nd }}$ & group & 247 & 211 & 167 & 102 \\
\hline $3^{r d}$ & group & 205 & 173 & 127 & 71 \\
\hline $4^{\text {th }}$ & group & 127 & 90 & 49 & 29 \\
\hline
\end{tabular}

\section{b Cardiovascular Mortality}

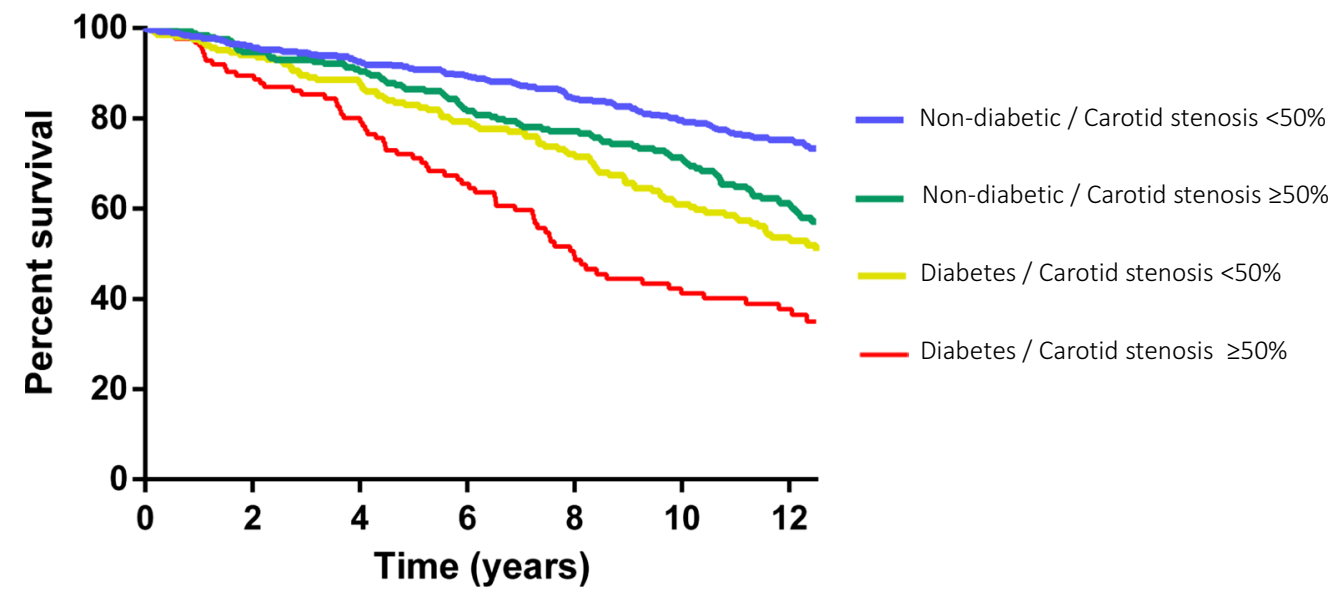

\begin{tabular}{|c|c|c|c|c|c|}
\hline \multicolumn{6}{|c|}{ No. at risk } \\
\hline $1^{\text {st }}$ & group & 486 & 428 & 369 & 244 \\
\hline $2^{\text {nd }}$ & group & 247 & 211 & 167 & 102 \\
\hline $3^{r d}$ & group & 205 & 173 & 127 & 71 \\
\hline $4^{\text {th }}$ & group & 127 & 90 & 49 & 29 \\
\hline
\end{tabular}

Fig. 1 Kaplan-Meier estimates of all-cause and cardiovascular mortality. During a median follow-up time of 11.81 years (IQR, 6.01-12.43) according to degree of carotid stenosis and prevalence of diabetes mellitus. Group 1 was defined by carotid narrowing of <50\% and without diabetes. Group 2 included patients with carotid narrowing $\geq 50 \%$ and without diabetes. Group 3 represented patients with carotid narrowing $<50 \%$ and diabetes, and group 4 patients with both carotid stenosis $\geq 50 \%$ and diabetes. Log-rank test was used for the overall comparison among groups. a KaplanMeier estimates of all-cause mortality. 549 (51.5\%) all-cause deaths were recorded. b Kaplan-Meier estimates of cardiovascular mortality. 367 (34\%) cardiovascular deaths were recorded

dipeptidyl-peptidase 4 (DPP-4) inhibitors have recently been introduced to the market and some of them have the capacity to significantly reduce cardiovascular events and even cardiovascular mortality [28, 29]. The findings sparked a debate in the community whether the very pronounced improvement of cardiovascular outcome can 
be attributed solely to the anti-diabetic effects of these drugs. Consequently, numerous post-trial studies are now evaluating the cardiovascular protective properties of these drugs [30]. In this context, it had been recently demonstrated that sitagliptin improves tissue characteristics of the carotid arterial wall [31].

Our data suggest that sonographic assessment of the degree of carotid stenosis at a single time-point can easily stratify diabetic patients in high and low risk groups for adverse cardiovascular outcome. Since the identification of high risk patients is of major importance for these trials, we believe that future drug related research could benefit from our findings.

\section{Limitations}

Although our results suggest a strong association between diabetes and outcome in patients with subclinical carotid atherosclerosis, we are aware of some limitations to our study. The study was initially designed to evaluate inflammatory biomarkers and we therefore have a lack of information about the dosage of insulin and the specific type of oral anti-diabetic drugs as well as baseline fasting plasma glucose levels. In addition, time-dependent factors, change of the therapeutic regimen and socio or environmental factors may influence the relationship between carotid stenosis, diabetes and long-term mortality. Due to advances in ultrasound technology, imaging has improved the assessment of plaques composition [32]. Since baseline investigations were performed in the early 2000s, we cannot provide data of the specific carotid plaque composition.

\section{Conclusion}

Diabetic patients with carotid stenosis $\geq 50 \%$ are at exceptional high risk for all-cause and cardiovascular death. Thus, routinely ultrasound investigation of the carotid arteries might be a valuable prognostic tool for patients with diabetes mellitus. However, further research is warranted to evaluate the clinical usefulness of ultrasound investigations of the carotid arteries as a screening tool in patients with diabetes mellitus and whether adjusted therapeutically intervention improves outcome in these patients.

\section{Abbreviations}

AGEs: advanced glycation end products; DPPI: dipeptidyl-peptidase 4 inhibitors; HbA1c: glycohemoglobin A1c; HR: hazard ratio; IQR: interquartile range; SGLT2: sodium-glucose co-transporter 2 inhibitors.

\section{Acknowledgements}

None.

\section{Authors' contributions}

FJM and GG did the statistical analyses. FJM and CJB wrote the paper. $\mathrm{MH}$ wrote part of the paper and contributed to the idea. EM and MS were responsible for the main conduct of the ICARAS study. All authors critically reviewed the manuscript and contributed to its content. All authors read and approved the final manuscript.

Funding

None.

Availability of data and materials

Patient data cannot be shared, because of protection of data privacy.

\section{Ethics approval and consent to participate}

The study protocol was in line with the Declaration of Helsinki and was approved by the institutional review board of the Medical University of Vienna. All patients gave their written informed consent.

Consent for publication

All authors of this paper have read and approved the final version submitted.

\section{Competing interests}

The authors declare that they have no competing interests.

\section{Author details}

${ }^{1}$ Department of Internal Medicine II, Division of Angiology, Medical University of Vienna, Vienna, Austria. ${ }^{2}$ Department of Internal Medicine II, Division of Cardiology, Medical University of Vienna, Vienna, Austria. ${ }^{3}$ Department of Laboratory Medicine, Medical University of Vienna, Währinger Gürtel 18-20, 1090 Vienna, Austria.

Received: 9 April 2019 Accepted: 9 July 2019

Published online: 12 July 2019

\section{References}

1. Chaudhuri J, Bains Y, Guha S, Kahn A, Hall D, Bose N, et al. The role of advanced glycation end products in aging and metabolic diseases: bridging association and causality. Cell Metab. 2018;28(3):337-52.

2. Shah MS, Brownlee M. Molecular and cellular mechanisms of cardiovascular disorders in diabetes. Circ Res. 2016;118(11):1808-29.

3. Vrsalovic M, Vucur K, Vrsalovic Presecki A, Fabijanic D, Milosevic M. Impact of diabetes on mortality in peripheral artery disease: a meta-analysis. Clin Cardiol. 2017:40(5):287-91.

4. Aronson D, Edelman ER. Coronary artery disease and diabetes mellitus. Cardiol Clin. 2014;32(3):439-55.

5. Hill MD. Stroke and diabetes mellitus. Handb Clin Neurol. 2014;126:167-74.

6. Menegazzo L, Poncina N, Albiero M, Menegolo M, Grego F, Avogaro A, et al. Diabetes modifies the relationships among carotid plaque calcification, composition and inflammation. Atherosclerosis. 2015;241(2):533-8.

7. Noh M, Kwon H, Jung CH, Kwon SU, Kim MS, Lee WJ, et al. Impact of diabetes duration and degree of carotid artery stenosis on major adverse cardiovascular events: a single-center, retrospective, observational cohort study. Cardiovasc Diabetol. 2017;16(1):74.

8. Schillinger M, Exner M, Mlekusch W, Sabeti S, Amighi J, Nikowitsch R, et al. Inflammation and carotid artery-risk for atherosclerosis study (ICARAS). Circulation. 2005;111(17):2203-9.

9. Grant EG, Benson CB, Moneta GL, Alexandrov AV, Baker JD, Bluth El, et al. Carotid artery stenosis: gray-scale and Doppler US diagnosissociety of radiologists in ultrasound consensus conference. Radiology. 2003;229(2):340-6.

10. Brott TG, Halperin JL, Abbara S, Bacharach JM, Barr JD, Bush RL, et al. ASA/ACCF/AHA/AANN/AANS/ACR/ASNR/CNS/SAIP/SCAI/SIR/SNIS/ SVM/SVS guideline on the management of patients with extracranial carotid and vertebral artery disease: executive summary: a report of the American College of Cardiology Foundation/American Heart Association Task Force on Practice Guidelines, and the American Stroke Association, American Association of Neuroscience Nurses, American Association of Neurological Surgeons, American College of Radiology, American Society of Neuroradiology, Congress of Neurological Surgeons, Society of Atherosclerosis Imaging and Prevention, Society for Cardiovascular Angiography and Interventions, Society of Interventional Radiology, Society of 
Neurolnterventional Surgery, Society for Vascular Medicine, and Society for Vascular Surgery. Developed in collaboration with the American Academy of Neurology and Society of Cardiovascular Computed Tomography. Catheter Cardiovasc Interv. 2011;81(1):E76-123.

11. World Health Organization. Report of the expert committee on the diagnosis and classification of diabetes mellitus. Diabetes Care. 1997;20(7):1183-97.

12. American Diabetes A. 2. Classification and diagnosis of diabetes: standards of medical care in diabetes-2019. Diabetes Care. 2019;42(Suppl 1):S13-28.

13. Pencina MJ, D'Agostino RB, D'Agostino RB, Vasan RS. Evaluating the added predictive ability of a new marker: from area under the ROC curve to reclassification and beyond. Stat Med. 2008;27(2):157-72.

14. Hernandez M, Lopez C, Real J, Valls J, Ortega-Martinez de Victoria E, Vazquez $\mathrm{F}$, et al. Preclinical carotid atherosclerosis in patients with latent autoimmune diabetes in adults (LADA), type 2 diabetes and classical type 1 diabetes. Cardiovasc Diabetol. 2017;16(1):94.

15. Naghavi M, Libby P, Falk E, Casscells SW, Litovsky S, Rumberger J, et al. From vulnerable plaque to vulnerable patient: a call for new definitions and risk assessment strategies: part I. Circulation. 2003;108(14):1664-72.

16. van Dijk AC, Donkel SJ, Zadi T, Sonneveld MAH, Schreuder F, Chohan MF, et al. Association between fibrinogen and fibrinogen gamma' and atherosclerotic plaque morphology and composition in symptomatic carotid artery stenosis: plaque-At-RISK study. Thromb Res. 2019;177:130-5.

17. Fernandez-Friera L, Fuster V, Lopez-Melgar B, Oliva B, Sanchez-Gonzalez $J$, Macias $A$, et al. Vascular inflammation in subclinical atherosclerosis detected by hybrid PET/MRI. J Am Coll Cardiol. 2019;73(12):1371-82.

18. Bots ML, Hoes AW, Koudstaal PJ, Hofman A, Grobbee DE. Common carotid intima-media thickness and risk of stroke and myocardial infarction: the Rotterdam Study. Circulation. 1997;96(5):1432-7.

19. Leary DH, Polak JF, Kronmal RA, Manolio TA, Burke GL, Wolfson SK. Carotid-artery intima and media thickness as a risk factor for myocardial infarction and stroke in older adults. Cardiovascular health study collaborative research group. N Engl J Med. 1999;340(1):14-22.

20. Lorenz MW, von Kegler S, Steinmetz H, Markus HS, Sitzer M. Carotid intima-media thickening indicates a higher vascular risk across a wide age range: prospective data from the carotid atherosclerosis progression study (CAPS). Stroke. 2006;37(1):87-92.

21. Lorenz MW, Price JF, Robertson C, Bots ML, Polak JF, Poppert H, et al. Carotid intima-media thickness progression and risk of vascular events in people with diabetes: results from the PROG-IMT collaboration. Diabetes Care. 2015;38(10):1921-9.

22. Katakami N, Mita T, Gosho M, Takahara M, Irie Y, Yasuda T, et al. Clinical utility of carotid ultrasonography in the prediction of cardiovascular events in patients with diabetes: a combined analysis of data obtained in five longitudinal studies. J Atheroscler Thromb. 2018;25(10):1053-66.

23. Nambi V, Chambless L, He M, Folsom AR, Mosley T, Boerwinkle E, et al. Common carotid artery intima-media thickness is as good as carotid intima-media thickness of all carotid artery segments in improving prediction of coronary heart disease risk in the Atherosclerosis Risk in Communities (ARIC) study. Eur Heart J. 2012;33(2):183-90.

24. Baldassarre D, Hamsten A, Veglia F, de Faire U, Humphries SE, Smit AJ, et al. Measurements of carotid intima-media thickness and of interadventitia common carotid diameter improve prediction of cardiovascular events: results of the IMPROVE (Carotid Intima Media Thickness [IMT] and IMT-Progression as Predictors of Vascular Events in a High Risk European Population) study. J Am Coll Cardiol. 2012;60(16):1489-99.

25. Jiang W, Nakayama Y, Sequeira JM, Quadros EV. Characterizing monoclonal antibodies to antigenic domains of TCbIR/CD320, the receptor for cellular uptake of transcobalamin-bound cobalamin. Drug Deliv. 2011;18(1):74-8.

26. Prati P, Vanuzzo D, Casaroli M, Di Chiara A, De Biasi F, Feruglio GA, et al. Prevalence and determinants of carotid atherosclerosis in a general population. Stroke. 1992;23(12):1705-11.

27. American Diabetes A. 10. Cardiovascular disease and risk management: standards of medical care in diabetes-2019. Diabetes Care. 2019;42(Suppl 1):S103-23.

28. Bell RM, Yellon DM. SGLT2 inhibitors: hypotheses on the mechanism of cardiovascular protection. Lancet Diabetes Endocrinol. 2018;6(6):435-7.

29. Xie W, Song X, Liu Z. Impact of dipeptidyl-peptidase 4 inhibitors on cardiovascular diseases. Vascul Pharmacol. 2018;109:17-26.

30. Fitchett D, Inzucchi SE, Cannon CP, McGuire DK, Scirica BM, Johansen OE, et al. Empagliflozin reduced mortality and hospitalization for heart failure across the spectrum of cardiovascular risk in the EMPA-REG OUTCOME Trial. Circulation. 2019;139(11):1384-95.

31. Katakami N, Mita T, Irie Y, Takahara M, Matsuoka TA, Gosho M, et al. Effect of sitagliptin on tissue characteristics of the carotid wall in patients with type 2 diabetes: a post hoc sub-analysis of the sitagliptin preventive study of intima-media thickness evaluation (SPIKE). Cardiovasc Diabetol. 2018;17(1):24.

32. ParkTH. Evaluation of carotid plaque using ultrasound imaging. J Cardiovasc Ultrasound. 2016;24(2):91-5.

\section{Publisher's Note}

Springer Nature remains neutral with regard to jurisdictional claims in published maps and institutional affiliations.
Ready to submit your research? Choose BMC and benefit from:

- fast, convenient online submission

- thorough peer review by experienced researchers in your field

- rapid publication on acceptance

- support for research data, including large and complex data types

- gold Open Access which fosters wider collaboration and increased citations

- maximum visibility for your research: over $100 \mathrm{M}$ website views per year

At BMC, research is always in progress.

Learn more biomedcentral.com/submissions 